\title{
The X-ray Spectrum of the Vela Pulsar Resolved with Chandra
}

\author{
G. G. Pavlov ${ }^{1}$, V. E. Zavlin ${ }^{2}$, D. Sanwal ${ }^{1}$, V. Burwitz ${ }^{2}$, and G. P. Garmire ${ }^{1}$
}

Received —

\footnotetext{
1 The Pennsylvania State University, 525 Davey Lab, University Park, PA 16802, USA; pavlov@astro.psu.edu

2 Max-Planck-Institut für Extraterrestrische Physik, D-85740 Garching, Germany; zavlin@xray.mpe.mpg.de
} 


\begin{abstract}
We report the results of the spectral analysis of two observations of the Vela pulsar with the Chandra X-ray observatory. The spectrum of the pulsar does not show statistically significant spectral lines in the observed $0.25-8.0 \mathrm{keV}$ band. Similar to middle-aged pulsars with detected thermal emission, the spectrum consists of two distinct components. The softer component can be modeled as a magnetic hydrogen atmosphere spectrum — for the pulsar magnetic field $B=3 \times 10^{12} \mathrm{G}$ and neutron star mass $M=1.4 M_{\odot}$ and radius $R^{\infty}=13 \mathrm{~km}$, we obtain $T_{\text {eff }}^{\infty}=0.68 \pm 0.03 \mathrm{MK}, L_{\text {bol }}^{\infty}=(2.6 \pm 0.2) \times 10^{32} \mathrm{erg} \mathrm{s}^{-1}$, $d=210 \pm 20$ pc (the effective temperature, bolometric luminosity, and radius are as measured by a distant observer). The effective temperature is lower than that predicted by standard neutron star cooling models. A standard blackbody fit gives $T^{\infty}=1.49 \pm 0.04 \mathrm{MK}, L_{\mathrm{bol}}^{\infty}=(1.5 \pm 0.4) \times 10^{32} d_{250}^{2} \mathrm{erg} \mathrm{s}^{-1}\left(d_{250}\right.$ is the distance in units of $250 \mathrm{pc}$ ); the blackbody temperature corresponds to a radius, $R^{\infty}=(2.1 \pm 0.2) d_{250} \mathrm{~km}$, much smaller than realistic neutron star radii. The harder component can be modeled as a power-law spectrum, with parameters depending on the model adopted for the soft component $-\gamma=1.5 \pm 0.3, L_{x}=(1.5 \pm 0.4) \times 10^{31} d_{250}^{2} \operatorname{erg~s}^{-1}$ and $\gamma=2.7 \pm 0.4$ $L_{x}=(4.2 \pm 0.6) \times 10^{31} d_{250}^{2} \mathrm{erg} \mathrm{s}^{-1}$ for the hydrogen atmosphere and blackbody soft component, respectively $\left(\gamma\right.$ is the photon index, $L_{x}$ is the luminosity in the $0.2-8 \mathrm{keV}$ band). The extrapolation of the power-law component of the former fit towards lower energies matches the optical flux at $\gamma \simeq 1.35-1.45$.
\end{abstract}

Subject headings: stars: neutron — pulsars: individual (PSR B0833-45) — X-rays: stars 


\section{Introduction}

X-ray observations of rotation-powered pulsars with the Einstein, EXOSAT and, particularly, ROSAT observatories have established (Ögelman 1995) that at least three middle-aged pulsars, PSR B0656+14, B1055-52 and Geminga, with characteristic ages of $10^{5}-10^{6} \mathrm{yr}$, show thermal soft X-ray radiation, with (blackbody) temperatures $0.5-1 \mathrm{MK}$, interpreted as emitted from the surfaces of cooling neutron stars (NSs). Investigation of this radiation is needed to understand the NS cooling history and to study the properties of the NS surface layers. Further observations with the ASCA observatory have confirmed that, as it had been suggested from the ROSAT data, the spectra of these pulsars at higher energies ( $\gtrsim 1.5-2 \mathrm{keV}$ ) are dominated by nonthermal, power-law components, with photon indices $\gamma \approx 1.3-1.6$, presumably generated in the NS magnetospheres (e.g., Wang et al. 1998). Studying the power-law components is important to understand the mechanisms of the multiwavelength (optical through gamma-rays) pulsar radiation.

In addition to these three pulsars (dubbed "The Three Musketeers" by Becker \& Trümper 1997), the (presumably) much younger Vela pulsar $(\tau=P /[2 \dot{P}]=11 \mathrm{kyr}$, $P=89.3 \mathrm{~ms}, \dot{E}=6.9 \times 10^{36} \mathrm{erg} \mathrm{s}^{-1}, B \sim 3 \times 10^{12} \mathrm{G}$ - see Taylor, Manchester, \& Lyne 1993 for other properties and references) has often been mentioned as a possible source of thermal radiation, based on softness of its spectrum (e.g., Ögelman 1995). Ögelman, Finley, \& Zimmermann (1993) analyzed the spectrum of the Vela pulsar detected with ROSAT. They obtained acceptable fits of the spectrum with a power law (PL) model with $\gamma \simeq 3.3$ and a blackbody $(\mathrm{BB})$ model of temperature $T^{\infty} \simeq 1.7 \mathrm{MK}$. The slope of the spectrum obtained in the PL fit is much steeper than typical nonthermal spectra of young and middle-aged pulsars. The temperature and luminosity inferred from the BB fit correspond to an effective radius of emitting region about $1.3 \mathrm{~km}$ at a distance $d=250 \mathrm{pc}$ (Cha, Sembach, \& Danks 1999). A two-component fit with a BB+PL model yielded a lower 
temperature, 1.3 MK, a larger radius, $3 \mathrm{~km}$ at $250 \mathrm{pc}$, and a surprisingly small photon index, $\gamma \sim 0.1$ (Ögelman 1995). Page, Shibanov, \& Zavlin (1996) fit the same spectrum with the NS hydrogen atmosphere models (Pavlov et al. 1995) and obtained a lower effective

temperature and a larger $R / d: T_{\text {eff }}^{\infty} \equiv g_{r} T_{\text {eff }} \simeq 0.8 \mathrm{MK}, d \simeq 300$ pc for $R^{\infty} \equiv g_{r}^{-1} R=13 \mathrm{~km}$ $\left(g_{r} \equiv\left[1-2 G M / R c^{2}\right]^{1 / 2}\right.$ is the gravitational redshift parameter).

However, the true spectrum of the observed soft X-ray pulsar radiation (not to mention the presence of two components) has remained elusive because angular resolution of the X-ray telescopes has been too low to separate the pulsar radiation from that of the bright pulsar-wind nebula (PWN) of about $2^{\prime}$ diameter around the pulsar (Harnden, Grant, \& Seward 1985; Markwardt \& Ögelman 1988). The subarcsecond resolution of the Chandra X-ray Observatory $(C X O)$ provides the first opportunity to resolve the pulsar spectrum. The high energy resolution of the CXO grating spectrometers allows one to address another important problem - if the soft component of the pulsar radiation indeed originates from the NS surface layers (an atmosphere), it may show spectral features (lines and photoionization edges) which can be used to investigate chemical composition, gravity and magnetic field of the surface layers.

In this Letter we present first results on the X-ray spectrum of the Vela pulsar obtained with $C X O$. We describe the observations and the spectral analysis in $\S 2$ and discuss some implications of the observed spectrum in $\S 3$.

\section{Observations and Spectral Analysis}

To investigate the low-energy part of the pulsar spectrum with high energy resolution, the spectrum dispersed with the Low-Energy Transmission Grating (LETG; see Brinkman et al. 2000) was imaged on the Spectroscopic Array of the High Resolution Camera 
(HRC-S; see Murray et al. 1997). The 25.6 ks observation (ObsID 127) was taken on 28 January 2000. For the analysis we used the Level 2 data files produced by the standard pipeline processing (v. R4CU5UPD11.1). The dispersed LETG spectrum (dispersion $1.148 \AA / \mathrm{mm})$ was extracted from a strip of a $3^{\prime \prime} 6(0.18 \mathrm{~mm})$ width in the cross-dispersion direction, which contains about $90 \%$ of source events dispersed at a given wavelength (see Chandra Proposers' Observatory Guide, v.3.0; CPOG hereafter). The zero-order and dispersed radiation from the PWN contaminates the dispersed pulsar spectrum at short wavelengths, $\lambda<5-6 \AA$, and the plate background dominates at $\lambda>60 \AA$. Since at $\lambda=50-60 \AA$ there are data only from the positive dispersed orders, we chose the range $\lambda=6-50 \AA(E=0.25-2.0 \mathrm{keV})$ for spectral analysis. The background was taken from boxes between $10^{\prime \prime}$ and $30^{\prime \prime}(0.49-1.46 \mathrm{~mm})$ from the center of the source spectrum in the cross-dispersion direction. To search for spectral lines in the dispersed pulsar spectrum, we binned the extracted source-plus-background and background spectra in $0.02 \AA$ bins (intrinsic resolution of the instrument in the chosen wavelength range is $\simeq 0.05 \AA$ ). We used groups of 10-20 sequent bins to estimate deviation in number of source counts in each bin of a given group from the mean value in the group. The maximum deviation, in both the positive and negative dispersed orders, was found to be at a $2.7 \sigma$ significance level. Therefore, we conclude that the LETG spectrum of the Vela pulsar shows no statistically significant spectral lines.

For further analysis, we removed contribution of the higher orders at longer wavelengths using a "bootstrap" method which works as follows (see also CPOG). One assumes that contamination from the higher orders can be neglected at the shortest wavelengths. The source counts in a short wavelength bin are used to deduce the corresponding high-order contributions of this bin to longer wavelengths with the aid of relative grating efficiencies of the higher orders. These contributions are subtracted, and the process is repeated working upwards in wavelength. Using this method, we obtained the first-order pulsar spectrum 
(91\% of the total dispersed data), with a source countrate $281 \pm 6 \mathrm{ks}^{-1}\left(312 \pm 6 \mathrm{ks}^{-1}\right.$ after correcting for the extraction efficiency) in the $0.25-2.0 \mathrm{keV}$ range

To examine various continuum models, we grouped the counts in 162 energy bins with at least 30 source counts per bin and fit the models making use of the first-order effective area ${ }^{3}$. We found that a simple PL model requires a large photon index $\gamma=4.1-4.3$ and an implausibly high hydrogen column density $n_{\mathrm{H}, 20} \equiv n_{\mathrm{H}} / 10^{20} \mathrm{~cm}^{-2}=9.1-9.3$; moreover, the best-fit model spectrum exceeds the observed one at $E \gtrsim 1.2 \mathrm{keV}$. The spectrum fits much better with thermal models (blackbody, NS hydrogen atmosphere), but the observed spectrum somewhat exceeds the model spectra at the highest energy channels (1.5-2.0 $\mathrm{keV}$ ), which indicates the presence of a second component with a harder spectrum.

To search for the harder component, we used the archived 37.0 ks observationf (ObsID 131; pipeline processing v. R4CU5UPD8.2) taken on 11-12 October 1999 with the Spectroscopic Array of the Advanced CCD Imaging Spectrometer (ACIS-S; Garmire et al. 2001) in Continuos Clocking (CC) mode which allows timing at the expense of one dimension of spatial resolution (along the CCD chip columns). This observation was carried out with the High Energy Transmission Grating (HETG), which, in principle, gives a high-resolution source spectrum at higher energies. However, the dispersed spectra, integrated along the chip columns in the $\mathrm{CC}$ mode, are severely contaminated by the chip background, which strongly complicates their analysis. Therefore, we use only the zero-order image (on the back-illuminated ACIS chip S3) which provides a pulsar spectrum with resolution of about $0.1-0.2 \mathrm{keV}$. The archival pipe-line processed data are not corrected properly for the satellite wobbling (dither) and for the Scientific Instrument Module motion, which smears the 1-D image. We applied these corrections making use of the

\footnotetext{
3 version of 31 Oct 2000; http://asc.harvard.edu/cal/Links/Letg/User/Hrc_QE/EA/

${ }^{4}$ Preliminary results of this observation were presented by Stage et al. (2000).
} 
algorithm kindly provided by Glenn Allen (see Zavlin et al. 2000 for details) and obtained one-dimensional (1-D) brightness distributions of the Vela pulsar and its PWN (bottom panel of Fig. 1). The upper panel of Figure 1 shows a 2-D image of this object obtained with the ACIS-S in the standard Timed Exposure mode (ObsID 128)]. This image is scaled and oriented in such a way that its horizontal axis is parallel to the 1-D image and its horizontal size is equal to the length of the 1-D distributions shown in the bottom panel. The 1-D profiles in different energy bands demonstrate that the pulsar is much brighter than the PWN background at $E \lesssim 1.5 \mathrm{keV}$, and it is discernible up to $8 \mathrm{keV}$, where the charged particle background takes over.

For the analysis of the pulsar spectrum, we extracted counts from a 1-D 2".5-wide aperture. The nonuniform (in both the total brightness and spectrum) PWN background was found by interpolating between the adjacent regions on both sides of the pulsar. The estimated source count rate is $288 \pm 4 \mathrm{ks}^{-1}$, or $320 \pm 5 \mathrm{ks}^{-1}$ after correcting for the $90 \%$ fraction of point source counts in the 1-D aperture chosen. Because of the dither, the pulsar and the PWN were imaged on two nodes of the chip (node 0 and node 1), which use different amplifiers and have different gains and responses. Therefore, we consider the two nodes as separate instruments while fitting the observed spectra. Since the ACIS response below $0.5 \mathrm{keV}$ is still poorly known, we discarded the corresponding counts.

We find that the ACIS spectrum of the pulsar does not fit with a one-component model, neither standard (BB, PL) nor a NS atmosphere spectrum, but it fits fairly well with two-component models (e.g., a PL plus a thermal component).

The spectral parameters obtained from fitting the separate HRC-S/LETG and ACIS-S

\footnotetext{
${ }^{5}$ Preliminary results of this observation were presented by Pavlov et al. (2000). See also http://chandra.harvard.edu/press/00_releases/press_060600vela.html
} 
spectra, as well as from the combined fit, are presented in Table 1. While fitting the LETG spectra, one cannot reliably find the parameters of the harder component, so we fix them at the values found from the ACIS-S fits. Similarly, the hydrogen column density $n_{\mathrm{H}}$ cannot be determined reliably from the ACIS-S spectra with low-energy counts discarded, so we fix $n_{\mathrm{H}}$ at the value found from the LETG fit. We see from Table 1 that the spectral parameters obtained from the separate fits are generally consistent with each other; the differences can be attributed to systematic errors caused by inaccuracies of the HRC/LETG and ACIS responses. The combined (LETG plus ACIS) fits (see Fig. 2) can be considered acceptable, in view of the current uncertainties of the instrument responses. Although we cannot completely exclude the possibility that the deviations from the continuous spectra might be due to some broad, shallow spectral features, the shortness of the exposures and the current knowledge of the instrument responses do not allow us to make definitive conclusions based on the data available.

\section{Discussion}

Thanks to the superb angular resolution of the CXO telescope, we are able to resolve the pulsar from its PWN and investigate its spectrum in the $0.25-8.0 \mathrm{keV}$ range. Our analysis proves that the pulsar soft X-ray emission, at $E \lesssim 1.8 \mathrm{keV}$, is indeed dominated by a soft thermal component, so that the Three Musketeers (see Introduction) are now joined by D'Artagnan - the Vela pulsar. The parameters of the soft thermal component are drastically different for the BB fitf $\left(T_{\mathrm{bb}}^{\infty}=1.4-1.5 \mathrm{MK}, R_{\mathrm{bb}}^{\infty}=[1.9-2.4] d_{250} \mathrm{~km}-\right.$ close to the values obtained by Ögelman et al. 1993 from the ROSAT spectrum) and the

\footnotetext{
${ }^{6} T_{\mathrm{bb}}^{\infty}$ and $R_{\mathrm{bb}}^{\infty}$ are the temperature and the radius given by the standard BB fits; we use the superscript ${ }^{\infty}$ by analogy with the case of atmosphere fits, to emphasize that in both cases the quantities are given as measured by a distant observer. It should be noted that $T_{\text {eff }}^{\infty}$
} 
hydrogen atmosphere fit $\left(T_{\mathrm{eff}}^{\infty}=0.65-0.71 \mathrm{MK}, R^{\infty}=[14-17] d_{250} \mathrm{~km}\right)$. For the BB model, one might assume that the observed radiation is emitted from small hot spots (e.g., polar caps heated by relativistic particles produced in the pulsar magnetoshere), whereas the $R / d$ ratio obtained in the $\mathrm{H}$ atmosphere fit implies radiation emitted from the entire NS surface, with a lower temperature.

Rigorously speaking, the surface (atmosphere) of a NS is not a black body, but a BB spectrum could mimic the spectrum of a heavy-element (e.g., iron) atmosphere if it is observed with low spectral resolution (Rajagopal, Romani, \& Miller 1997). However, a lack of significant spectral lines in the LETG spectrum of the Vela pulsar hints that there are no heavy elements in the radiating atmosphere layers (although the exposure was too short to take full advantage of the high spectral resolution).

Because of the enormous NS gravity, the outer layers of the NS atmosphere should be comprised of the lightest element present. If, in the absence of $\mathrm{H}$, the NS has a He atmosphere, we still expect spectral features in the observed range if the magnetic field is as strong as predicted by the standard radio pulsar models, $B \sim 3 \times 10^{12} \mathrm{G}$. In such a field the He atmosphere is not completely ionized even at $T \sim 1 \mathrm{MK}$, mainly because of the large increase of the ionization potentials (Ruder et al. 1994): $I \simeq 0.63 \mathrm{keV}$ for the one-electron He ion $(\approx 0.5 \mathrm{keV}$ with account for the gravitational redshift). The fact that we see neither a photoionization edge around $E \sim 0.5 \mathrm{keV}$ nor spectral lines at somewhat lower energies suggests that there is no He in the atmosphere. On the other hand, if even a small amount of $\mathrm{H}$ is present in the surface layers $\left(\sim 10^{12}-10^{13} \mathrm{~g}\right.$ of $\mathrm{H}$ over the NS surface would be enough to hide heavier elements completely), the hydrogen, with its lower ionization potential, $I \simeq 0.23 \mathrm{keV}$, should be strongly ionizied, and if even some neutral

and $R^{\infty} / d$ very weakly depend on the input parameters of the hydrogen atmosphere models (Zavlin, Pavlov, \& Trümper 1998). 
fraction is present, it will not show spectral features at $E \gtrsim 0.17 \mathrm{keV}$. Thus, the featureless spectrum we observe is consistent with the hypothesis of a hydrogen NS atmosphere. The observed part of the (continuum) spectrum of such an atmosphere decreases with $E$ slower than the ideal Wien spectrum because the radiation at higher energies comes from hotter layers (the $\mathrm{H}$ opacity strongly decreases with frequency). As a result, BB fits to the $\mathrm{H}$ atmosphere spectra give a temperature higher than the true effective temperature and an area smaller than the true emitting area (e.g., Pavlov et al. 1995). If we adopt the $\mathrm{H}$ atmosphere hypothesis, the effective temperature of the Vela pulsar is below the predictions of the so-called standard models of the NS cooling (e.g., Tsuruta 1998), if even the pulsar is a factor of 2-3 older than its characteristic age (Lyne et al. 1996).

An important result of our analysis is the detection of the hard spectral component. Because of the small number of high-energy photons detected, both the PL and thermal fits of this component are formally acceptable. However, the thermal fits yield an implausibly small size, $\sim 10 \mathrm{~m}$, and a very high temperature, $\sim 10 \mathrm{MK}$, of the emitting region, and extrapolation of the model spectrum to lower and higher energies is inconsistent with optical and hard-X-ray observations. Therefore, we favor the nonthermal interpretation of the hard component - a PL $(\gamma=1.2-1.8$ if the $\mathrm{H}$ atmosphere model is adopted for the soft component) which dominates at $E \gtrsim 1.8 \mathrm{keV}$. With this interpretation, the overall X-ray spectrum of the the Vela pulsar is quite similar to those of the Three Musketeers, which show the nonthermal component dominating above $1.5-2.0 \mathrm{keV}$, with $\gamma \approx 1.3-1.6$. It is interesting that the ratio of the nonthermal X-ray luminosity (estimated as if the radiation were isotropic, $\left.L_{x}=4 \pi d^{2} F_{x}\right)$ to the spin-down energy loss, $L_{x} / \dot{E}=(1.7-2.6) \times 10^{-6}$ in the $0.2-8.0 \mathrm{keV}$ range, or $(2.1-3.4) \times 10^{-6}$ in the ROSAT range $0.1-2.4 \mathrm{keV}$, is much smaller than $L_{x} / \dot{E} \sim 10^{-3}$ for majority of X-ray detected pulsars (Becker \& Trümper 1997). Thus, luckily for us, the magnetosphere of the Vela pulsar is very underluminous in the X-ray range - if its $L_{x} / \dot{E}$ were as high as for other pulsars, the thermal radiation would be 
undetectable in the phase-integrated spectrum.

Since the Vela pulsar has been detected in the optical and gamma-ray ranges, where its radiation is certainly nonthermal, it is illuminating to compare its X-ray nonthermal spectrum with those in the other ranges. Figure 3 shows the energy flux spectrum of the pulsar from optical to gamma-rays. We see that extrapolation of the PL spectrum obtained in the atmosphere+PL fit matches fairly well with the optical and hard-X-ray (soft- $\gamma$-ray) time-averaged fluxes. On the contrary, the slope of the PL component in the BB+PL fit is too steep to be consistent with the optical and gamma-ray data, which is an additional argument in favor of the $\mathrm{H}$ atmosphere model. Moreover, if we adopt the hypothesis that the spectrum has the same slope in the optical through X-ray range, we can include the optical points in the fit for the PL component, which constrains the photon index and the power-law normalization very tightly: $\gamma=1.35-1.45$,

In the present paper we analyze only the phase-integrated spectra. Energy-integrated light curves, with at least three peaks per period, have been observed with the CXO HRC-I detector (Pavlov et al. 2000; Helfand, Gotthelf, \& Halpern 2000). In fact, the phase-resolved spectral analysis, or energy-resolved timing, can provide much more information about the pulsar. For instance, we should expect that the pulsed fraction, pulse shapes (and, perhaps, even the number of peaks per period) are quite different at energies below and above $1.8 \mathrm{keV}$ because of the different emission mechanisms. However, because of the low pulsed fraction and complicated light curves, such an analysis would require an order of magnitude more pulsar photons than 16,000 photons collected in the described observations.

We are grateful to Leisa Townsley and George Chartas for the useful advice on the analysis of ACIS data. Our special thanks are due to Glenn Allen, who provided the algorithm to correct the event times and coordinates in CC mode. We thank Gottfried Kanbach and Volker Schönfelder for providing the EGRET and COMPTEL fluxes in a 
digital form. GGP, VEZ and DS are thankful to the Institute for Theoretical Physics (UCSB), where a part of this work was done. This research was supported by NASA grants NAG5-7017 and NAS8-38252, SAO grant GO1-2071X, NSF grant PHY99-07949, and DLR (Deutsches Zentrum für Luft- und Raumfahrt) grant 50.OO.9501.9. 


\section{REFERENCES}

Becker, W., \& Trümper, J. 1997, A\&A, 326, 682

Brinkman, A. C., et al. 2000, ApJ, 530, L111

CPOG: Chandra Proposers' Observatory Guide, v.3.0, 2000, Chandra X-ray Center (Cambridge, Massachusetts) ( http://asc.harvard.edu/)

Cha, A., Sembach, K. R.,\& Danks, A. C. 1999, ApJ, 515, L25

Garmire, G. P., et al. 2001, in preparation

Harnden, F. R., Grant, P. D., Seward, F. D., \& Kahn, S. M. 1985, ApJ, 299, 828

Helfand, D. J., Gotthelf, E. F., \& Halpern, J. P. 2000, astro-ph/0007310

Kanbach, G., et al. 1994, A\&A, 289, 855

Lyne, A. G., Pritchard, R. S., Graham-Smith, F., \& Camilo, F. 1996, Nature, 381, 497

Markwardt, C. B., \& Ögelman, H. 1998, Memorie della Societa Astronomia Italiana, 69, 927

Murray, S. S., et al. 1997, SPIE, 3114, 11

Nasuti, F. P., Mignani, R., Caraveo, P. A., \& Bignami, G. F. 1997, A\&A, 323, 839

Ögelman, H. 1995, in The Lives of the Neutron Stars, eds. M. A. Alpar, Ü. Kiziloğlu \& J. van Paradijs (Kluwer: Dordrecht), p. 101

Ögelman, H., Finley, J. P., \& Zimmerman, H.-U. 1993, Nature, 361, 136

Page, D., Shibanov, Yu. A., \& Zavlin, V. E. 1996, MPE Reports, 263, 173 
Pavlov, G. G., Shibanov, Yu. A., Zavlin, V. E., \& Meyer, R. D. 1995, in The Lives of the Neutron Stars, eds. M. A. Alpar, Ü. Kiziloğlu \& J. van Paradijs (Kluwer: Dordrecht), p. 71

Pavlov, G. G., Sanwal, D., Garmire, G. P., Zavlin, V. E., Burwitz, V., \& Dodson, R. G. 2000, AAS Meeting 196, \#37.04

Rajagopal, M., Romani, R. W., \& Miller, M. C. 1997, ApJ, 479, 347

Schönfelder et al. 2000, A\&AS, 143, 145

Stage, M. D., Marshall, H. L., Schultz, N. S., \& Canizares, C. R. 2000, HEAD Meeting 32, \#33.05

Strickman, M. S., et al. 1996, ApJ, 460, 735

Taylor, J. H., Manchester, R. N., \& Lyne, A. G. 1993, ApJS, 88, 529

Tsuruta, S. 1998, Phys. Rep., 292, 1

Wang, F. Y.-H., Ruderman, M., Halpern, J. P., \& Zhu, T. 1998, ApJ, 498, 373

Zavlin, V. E., Pavlov, G. G., \& Trümper, J. 1998, A\&A, 331, 821

Zavlin, V. E., Pavlov, G. G., Sanwal, D., \& Trümper J. 2000, ApJ, 540, L25 
Fig. 1.- Bottom: One-dimensional profiles of the Vela pulsar and its PWN in different energy bands. One pixel is equal to 0".492. Top: $98^{\prime \prime} \times 70^{\prime \prime}$ ACIS-S3 image of the Vela pulsar and the PWN, with the horizontal axis parallel to the orientation of the 1-D image.

Fig. 2.- Combined fit of the HRC-S/LETG and ACIS-S3 count rate spectra with the two-component (magnetic hydrogen NS atmosphere plus power law) model. The dotted and dashed lines show the contributions of the thermal and nonthermal components. The best-fit parameters are given in Table 1.

Fig. 3.- Multiwavelength $(1 \mathrm{eV}-10 \mathrm{GeV})$ energy spectrum of the Vela pulsar. In addition to the CXO spectrum from this work, data shown include results from optical (Nasuti et al. 1997; U,B,V fluxes, without de-reddening), OSSE (Strickman et al. 1996), COMPTEL (Schönfelder et al. 2000) and EGRET (Kanbach et al. 1994) observations. The solid line shows a two-component (NS atmosphere plus power-law) fit to the observed $C X O$ 0.25-8 $\mathrm{keV}$ spectrum with spectral parameters $T_{\text {eff }}^{\infty}=0.68 \mathrm{MK}, R^{\infty}=15.5 d_{250} \mathrm{~km}, \gamma=1.4$, $\mathcal{N}=2.3 \times 10^{-4}, n_{\mathrm{H}, 20}=3.2$. The dotted lines correspond to same model spectrum corrected for the interstellar absorption and extrapolated to lower and higher energies. The dash-dot lines show the extrapolated optical and EUV spectra as absorbed by the interstellar matter. The thin solid lines demonstrate the uncertainty of the inferred power-law component. A typical error of the thermal component is shown by the vertical errorbar at the maximum of the spectrum. 
Table 1: Parameters of the two-component spectral fits, with $1 \sigma$ uncertainties.

\begin{tabular}{|c|c|c|c|}
\hline Parameter & HRC-S/LETG & ACIS-S & combined \\
\hline \multicolumn{4}{|c|}{ Blackbody + Power law } \\
\hline$T_{\mathrm{bb}}^{\infty}$ & $1.47 \pm 0.06$ & $1.51 \pm 0.03$ & $1.49 \pm 0.04$ \\
\hline$R_{\mathrm{bb}}^{\infty}$ & $2.2 \pm 0.3$ & $2.0 \pm 0.2$ & $2.1 \pm 0.2$ \\
\hline$L_{\mathrm{bol}}^{\infty}$ & $1.6 \pm 0.3$ & $1.4 \pm 0.3$ & $1.5 \pm 0.4$ \\
\hline$\gamma$ & $(2.2)$ & $2.2 \pm 0.4$ & $2.7 \pm 0.4$ \\
\hline $\mathcal{N}$ & $(6.0)$ & $6.0 \pm 1.3$ & $10.7 \pm 1.0$ \\
\hline$L_{x, \mathrm{pl}}$ & $(1.8)$ & $2.2 \pm 0.3$ & $4.2 \pm 0.6$ \\
\hline$n_{\mathrm{H}, 20}$ & $1.7 \pm 0.3$ & $(1.7)$ & $2.2 \pm 0.3$ \\
\hline$\chi_{\nu}^{2}$ [d.o.f. $]$ & $1.0[159]$ & $1.3[113]$ & $1.1[274]$ \\
\hline \multicolumn{4}{|c|}{$\mathrm{H}$ atmosphere + Power law } \\
\hline$T_{\mathrm{eff}}^{\infty}$ & $0.67 \pm 0.03$ & $0.69 \pm 0.03$ & $0.68 \pm 0.03$ \\
\hline$d$ & $200 \pm 25$ & $220 \pm 20$ & $210 \pm 20$ \\
\hline$L_{\mathrm{bol}}^{\infty}$ & $3.9 \pm 0.3$ & $3.7 \pm 0.3$ & $3.8 \pm 0.3$ \\
\hline$\gamma$ & $(1.4)$ & $1.4 \pm 0.3$ & $1.5 \pm 0.3$ \\
\hline $\mathcal{N}$ & $(2.3)$ & $2.3 \pm 0.7$ & $2.5 \pm 0.6$ \\
\hline$L_{x, \mathrm{pl}}$ & $(0.5)$ & $1.3 \pm 0.4$ & $1.5 \pm 0.4$ \\
\hline$n_{\mathrm{H}, 20}$ & $3.0 \pm 0.3$ & $(3.0)$ & $3.3 \pm 0.3$ \\
\hline$\chi_{\nu}^{2}$ [d.o.f. $]$ & $0.7[159]$ & $1.2[113]$ & $1.0[274]$ \\
\hline
\end{tabular}

Note. - The values in parentheses were fixed during fitting. The temperatures $T_{\mathrm{bb}}^{\infty}$ and $T_{\text {eff }}^{\infty}$ are in million kelvins $(\mathrm{MK})$. The radius $R_{\mathrm{bb}}^{\infty}(\mathrm{in} \mathrm{km})$ and the luminosities $L_{\mathrm{bol}}^{\infty}$ and $L_{x, \mathrm{pl}}\left(\right.$ in $10^{32} \mathrm{erg} \mathrm{s}^{-1}$ ) are related to $d=250 \mathrm{pc}$. The nonthermal luminosities $L_{x, \mathrm{pl}}$ are in the bands $0.2-2,0.5-8$, and $0.2-8 \mathrm{keV}$ for the LETG, ACIS-S, and combined spectra, respectively. $\gamma$ and $\mathcal{N}$ are the photon index and normalization constant of the power-law component: $\mathrm{d} N / \mathrm{d} E=\mathcal{N} E^{-\gamma}, E$ in $\mathrm{keV}, \mathcal{N}$ in $10^{-4}$ photons $/\left(\mathrm{cm}^{2} \mathrm{~s} \mathrm{keV}\right)$. The hydrogen atmosphere models were calculated for $B=3 \times 10^{12} \mathrm{G}, M=1.4 M_{\odot}, R=10 \mathrm{~km}\left(g_{r}=0.766\right.$, $\left.R^{\infty}=13.05 \mathrm{~km}\right)$. 


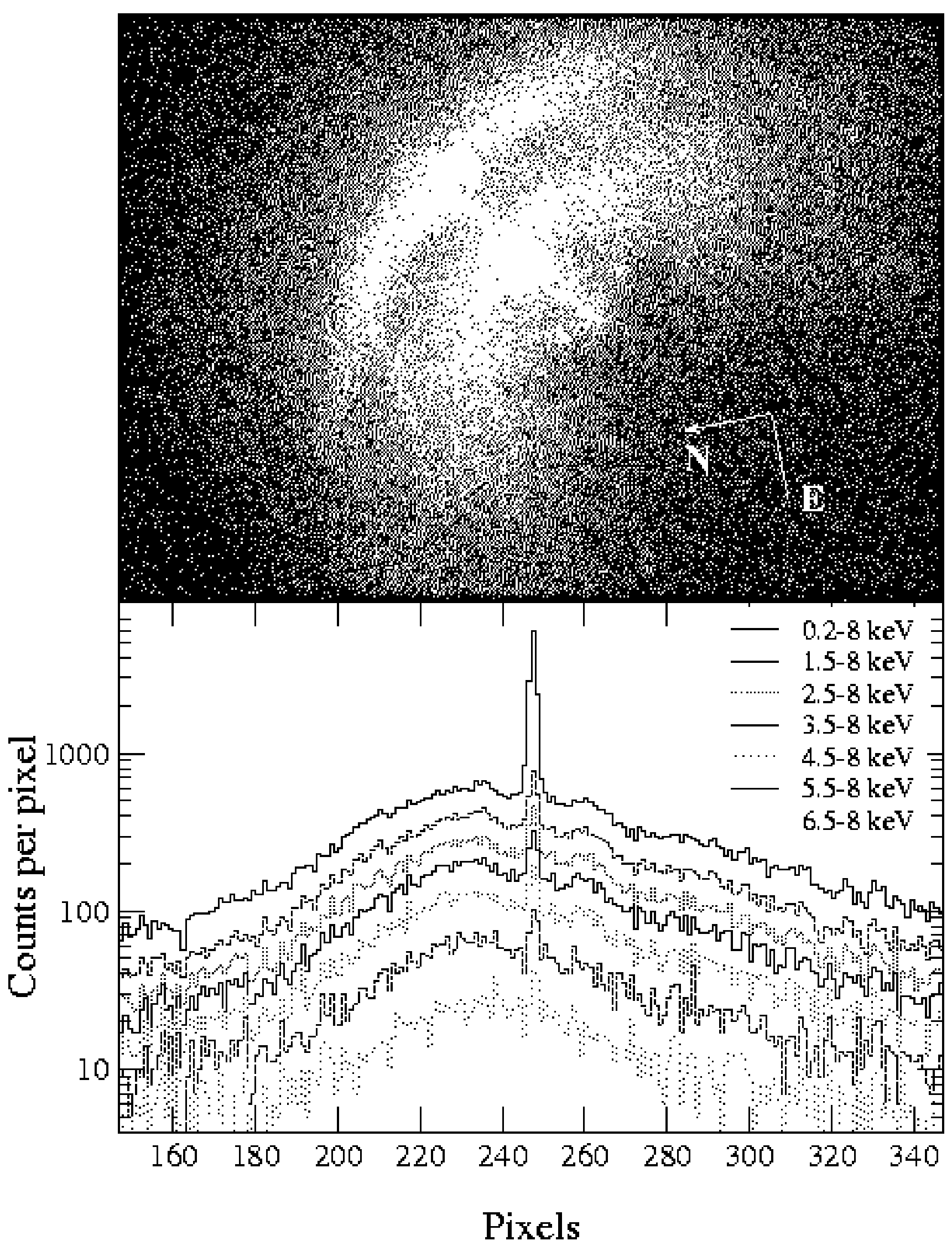



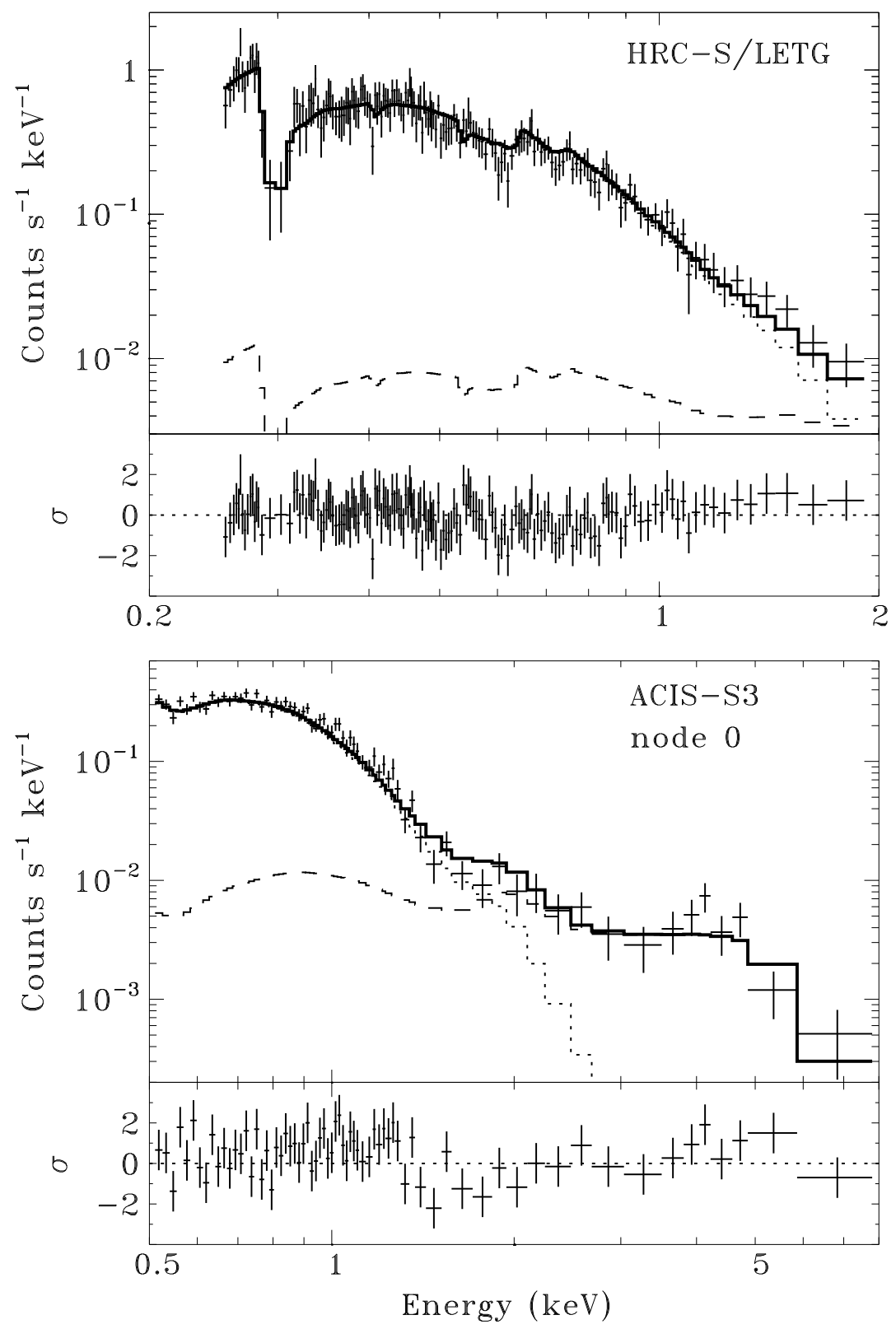


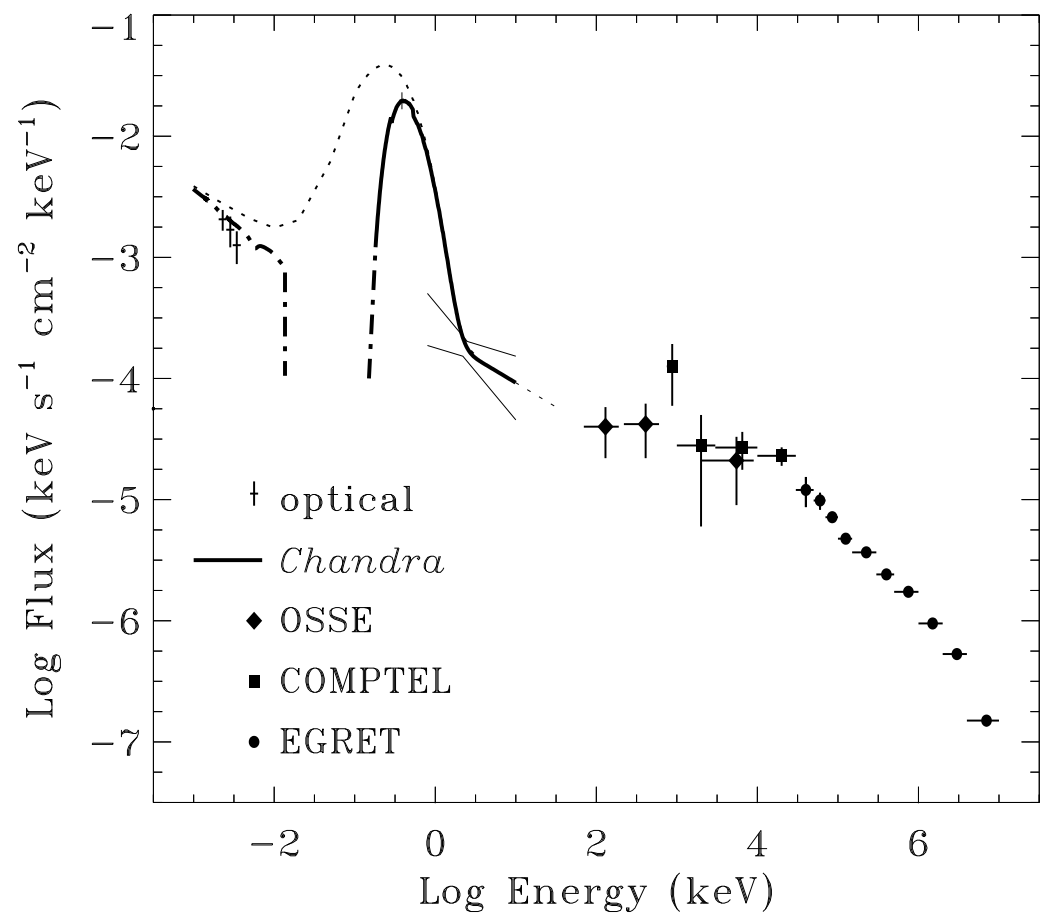

